\title{
DYNAMIC AXLE LOAD OF AN AUTOMOTIVE VEHICLE WHEN DRIVEN ON A MOBILE MEASUREMENT PLATFORM
}

\author{
C. JAGIEŁOWICZ-RYZNAR \\ Department of Applied Mechanics and Robotics \\ Faculty of Mechanical Engineering and Aeronautics \\ Rzeszow University of Technology \\ al. Powstańców Warszawy 12, 35-959 Rzeszów, POLAND \\ E-mail: cjr@prz.edu.pl
}

\begin{abstract}
An analysis of the dynamic axle load of an automotive vehicle (AV) when it is driven on a mobile measurement platform is presented in this paper. During the ride, the time characteristic of the dynamic force $\mathrm{N}(\mathrm{t})$, acting on the axle, was recorded. The effect of the vehicle axle mass on the maximum dynamic force value and the dynamic coefficient were studied. On this basis it was attempted to calculate the total vehicle's weight. Conclusions concerning the dynamic loads of the vehicle axles in relation to the reduced axle mass, were drawn. The optimal axle mass value, for which the dynamic coefficient reaches a minimum, was calculated.
\end{abstract}

Key words: mechanic vibrations, dynamic load, automotive vehicle axle.

\section{Introduction}

In the time of intensive development of the automotive industry, the maintenance and the development of road infrastructure are very big challenges. The road network is increasingly exposed to surface destruction, as a result of excessive and improper operation, mainly by multi-tone and -axle automotive vehicles. One of the questions related to exploitation of public roads by heavy automotive vehicles is the measurement of the axle pressure force on the roadway. Vehicles in which the pressure force of a single axle does not exceed the limit values (Journal of Laws of the Republic of Poland, 2012), taking into consideration also the type of road, were allowed to the traffic flow by the traffic law. The regulation of the Ministry of Infrastructure and Development (Traffic Law Act, 1997) enforces a limitation of axle pressure force from 8 or 10 tons on some road sections.

To verify whether the limitation is observed, the mass of heaviest vehicles is controlled by measuring the pressure force of particular axles and the whole mass as well. There are two types of measurements. The first one is a static measurement. The vehicle is stopped, directed to the control place, where the proper measuring and other operations are conducted. These activities involve various inconveniences, such as a waste of time and difficulties in the traffic. The other one consists in using a stationary measuring device permanently embedded in the lane. This way of control is associated with a complete lack of mobility and limits the measurement possibilities on other sections of the road. The static measurement can be supplemented by a dynamic measurement using the fact that during motion individual parts of a vehicle (especially the body) vibrate vertically. For each vehicle, these vibrations are forced, kinematically excited and due to road unevenness.

Under ideal conditions, the vehicle does not vibrate or vibrations are very small. To induce vibrations of a car body (which will not be dangerous) it is suggested to use a mobile measuring platform (MMP), in the shape of an inclined plane with a small angle (Fig.1). It will be possible to set the MMP on any traffic lane in any road section. A patent application for the platform has been filed at the Patent Office (patent application No. P.402614). The kinematically excited vibrations mainly depend on the vehicle weight, suspension stiffness, stiffness of the tire, damping in the shock absorbers and tires. 


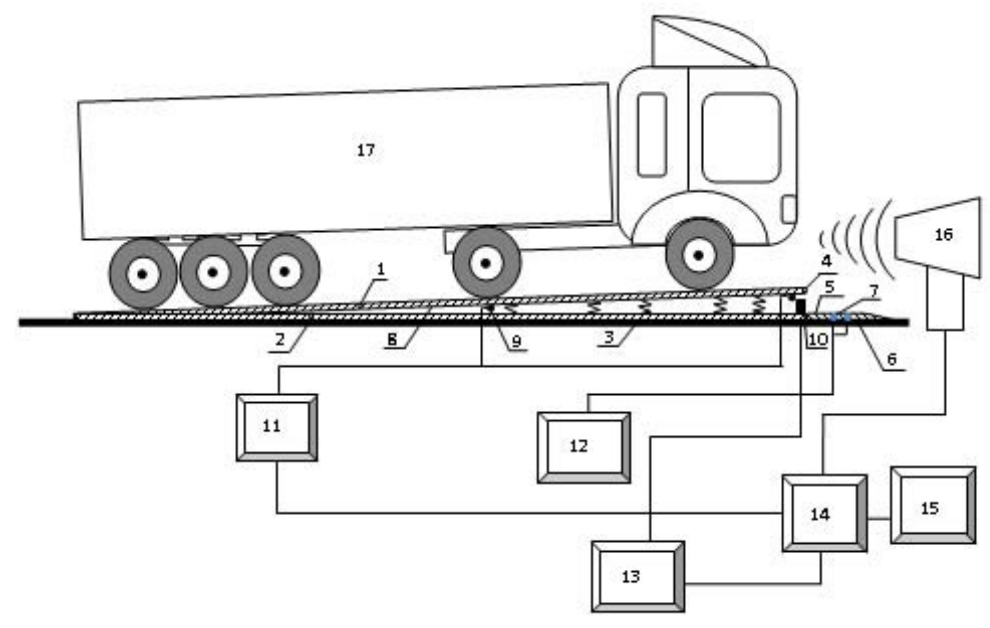

Fig.1. Schema of the MMP with an automotive vehicle driven on it (Jagiełowicz-Ryznar, 2013).

Legend for Fig.1:

1- ramp plate

2- support plate

3- spring elements

4- slope edge of the ramp plate

5- projecting zone of the support plate

6- lower surface of the support plate

7- vibration probes

8- ramp plate plane

9- measuring probes

10-measuring probe

$11,12,13$ - meters

14- programmed processor

15- monitor

16- horizontal speed sensor of an approaching vehicle

17-vehicle

\section{Model}

Dynamic loads that act on the vehicle axle when the AV is driven on the MMP were analyzed. During the ride the time characteristics of the dynamic force acting on the axle were recorded. On the basis of the $\mathrm{N}(\mathrm{t})$ characteristics the maximum dynamic load of the vehicle axle, as well as that of the road under real conditions, i.e., during normal operation, can be determined. The recorded $N(t)$ characteristics render it possible to determine some vehicle dynamic parameters such as speed, AV weight, shock absorber damping, natural frequencies and other parameters by means of using an intelligent computing system. This work neither resolves all the problems nor answers all the questions. It is merely an initial attempt to deal with the issue. Work on this area (with a model as well as parameter values) is continued and the results will be published.

The dynamic model of the system under analysis is shown in Fig.2. 


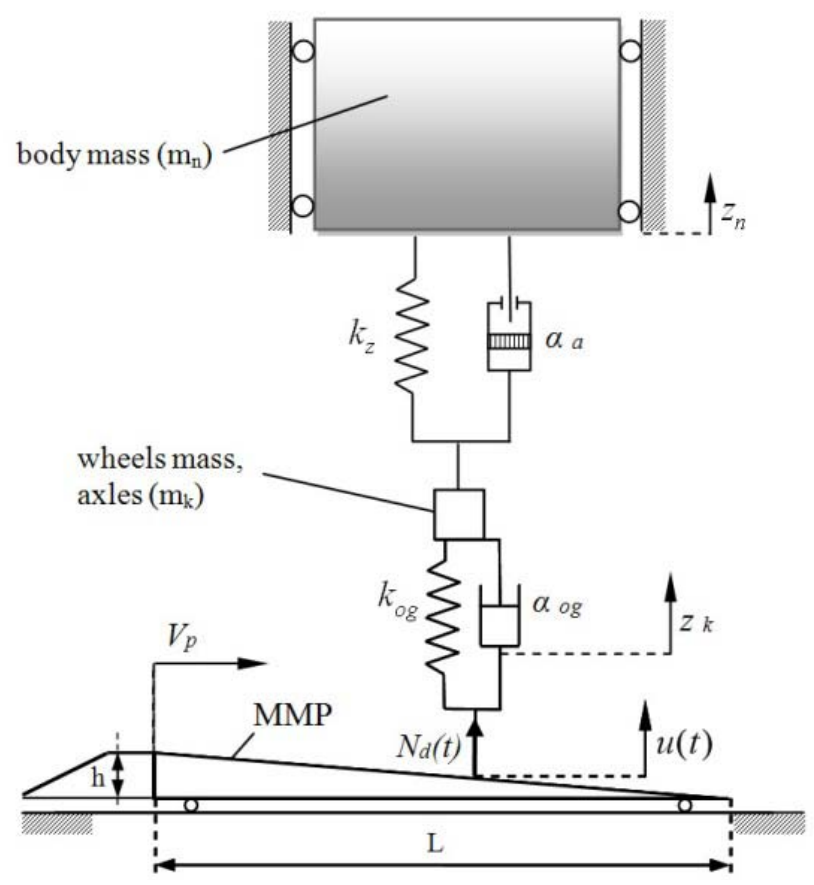

Fig.2. AV dynamic model.

The system has got two degrees of freedom associated with masses:

- the mass of body parts corresponding to the load of the axle under analysis,

- $\quad$ the mass reduced to this axle.

Forced vibrations are generated by the MMP when the AV is driven on it. (Fig.1)

The dynamic equations describing the vibrations of the system have the form (1)

$$
\begin{aligned}
& m_{n} \cdot \ddot{z}_{n}+k_{z}\left(z_{n}-z_{k}\right)+\alpha_{a}\left(\dot{z}_{n}-\dot{z}_{k}\right)=0, \\
& m_{k} \cdot \ddot{z}_{k}+k_{z}\left(z_{k}-z_{n}\right)+\alpha_{a}\left(\dot{z}_{k}-\dot{z}_{n}\right)+k_{\text {og }} \cdot z_{k}+\alpha_{o g} \cdot \dot{z}_{k}=k_{o g} \cdot u+\alpha_{o g} \cdot \dot{u} .
\end{aligned}
$$

Initial conditions are zero. Vibrations coming from the road were not taken into consideration. The function $u(t)$ describes the kinematically excited vibrations of axles and the body (assuming that the AV $V_{p}$ speed is constant) and has the form (Jagiełowicz-Ryznar, 2013)

$$
u(t)=\frac{h}{L} \cdot V_{p} \cdot t ; \quad t \in<0 ; \quad t_{p}=\frac{L}{V_{p}}>
$$

Formulas (2.1) and (2.2) show that the force $P_{w}(t)$ has the shape of a trapezoidal impulse with time $t_{p}$ and value $S_{w}$.

$$
P_{w}=V_{u} \cdot\left(\alpha_{o g}+k_{o g} \cdot t\right)
$$

where

$$
V_{u}=\frac{h}{L} \cdot V_{p},
$$




$$
S_{w}=h \cdot\left(\alpha_{o g}+\frac{k_{o g} \cdot L}{2 \cdot V_{p}}\right)
$$

The dynamic force $N_{d}(t)$ acting on the AV axle is described by formula (2.5)

$$
N_{d}(t)=k_{o g} \cdot\left(u-z_{k}\right)+\alpha_{o g} \cdot\left(V_{u}-V_{k}\right)
$$

where $\quad V_{k}=\frac{d z_{k}}{d t}$.

On the other hand, the dynamic coefficient $\eta_{d}$

$$
\eta_{d}=\frac{N_{d, \max }}{g \cdot\left(m_{n}+m_{k}\right)}
$$

The free vibration frequency $\omega_{o, i}$, the coefficients of normal vibration modes $\mu_{i}$, and the orthogonal coefficient $\mu$ of the system are presented by formulas (2.7), (2.8) and (2.9) (Kaliski, 1966)

$$
\omega_{o, i}=\sqrt{\frac{m_{k} \cdot k_{z}+m_{n} \cdot\left(k_{o g}+k_{z}\right) \mp \sqrt{\left[m_{k} \cdot k_{z}+m_{n} \cdot\left(k_{o g}+k_{z}\right)\right]^{2}-4 m_{k} \cdot m_{n} \cdot k_{o g} \cdot k_{z}}}{2 m_{k} \cdot m_{n}}},
$$

for $i=1$, sign (-); for $i=2$, sign (+);

$$
\begin{aligned}
& \mu_{i}=\frac{1}{1-\frac{m_{n}}{k_{z}} \cdot \omega_{o, i}^{2}} ; \quad i=1,2 . \\
& \mu=1+\mu_{1} \cdot \mu_{2} .
\end{aligned}
$$

\section{Calculation results}

Initially, 8 simulations were carried out for the following values of the axle mass (wheels) $m_{k}=1(0) ; 50 ; 100 ; 200 ; 500 ; 1000 ; 2500 ;[\mathrm{kg}]$

Other data:

$m_{n}=10000[\mathrm{~kg}] ; \quad k_{z}=3.5 \cdot 10^{6}[\mathrm{~N} / \mathrm{m}] ; \quad k_{\text {og }}=9 \cdot 10^{6}[\mathrm{~N} / \mathrm{m}] ; \quad \alpha_{a}=2 \cdot 10^{4}[\mathrm{Ns} / \mathrm{m}] ; \quad \alpha_{\text {og }}=2[\mathrm{Ns} / \mathrm{m}] ;$ MMP dimensions: $L=10[\mathrm{~m}] ; h=2[\mathrm{~cm}] ; V_{p}=20[\mathrm{~m} / \mathrm{s}]-$ - speed of the vehicle;

The calculation results are shown in diagrams $N_{d}(t)$ and $\eta_{d}(t)$ (Figs: 3a, 3b, 3c, 3d, 3e, 3f, 3g, $3 \mathrm{~h}$ ) and Tabs 1 and 2. On the basis of graphs (3a-h), graphs for $N_{d, \max }\left(\lg m_{k}\right)$ and $\eta_{d}\left(\lg m_{k}\right)$ in a comparatively large range $m_{k} \in<0 ; 2500>$ were prepared (Fig.4).

The simulations for $m_{k}=0$ and $m_{k}=1$ were performed for computation purposes. 
a)
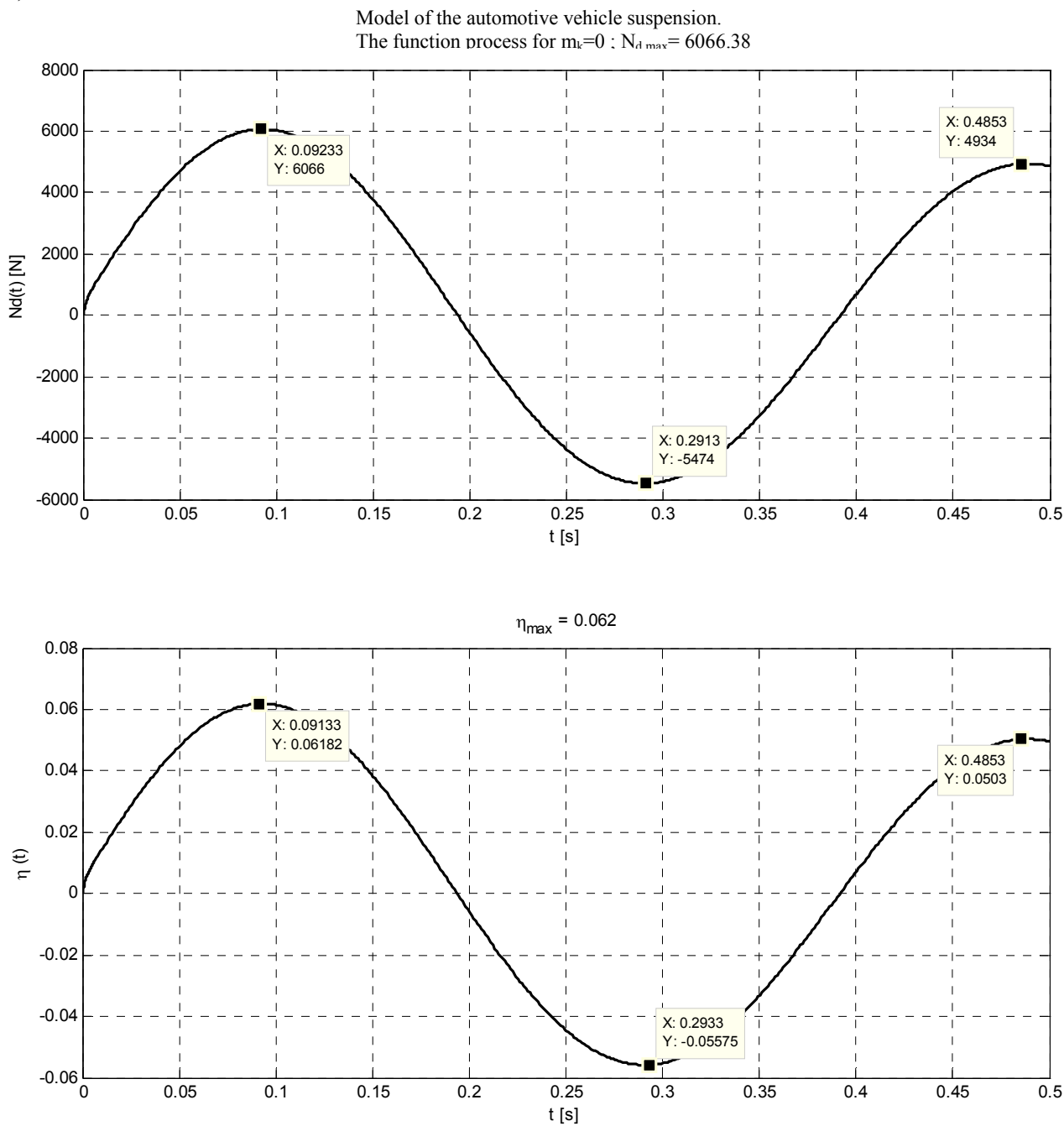

b)

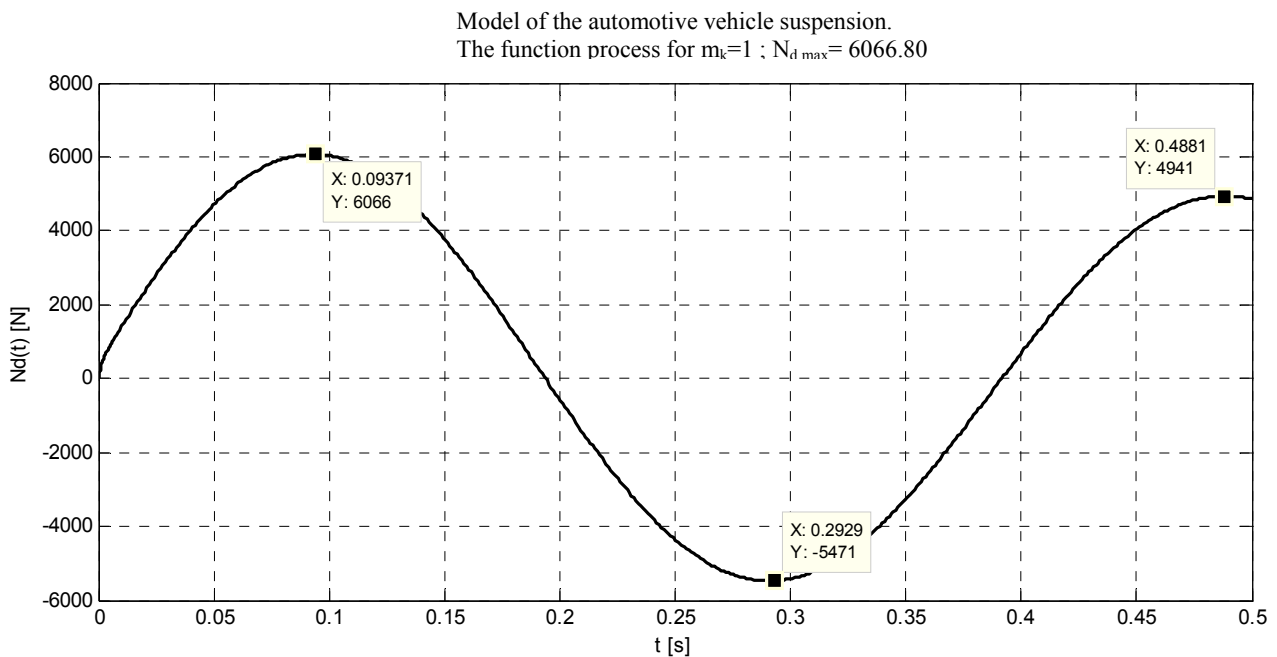




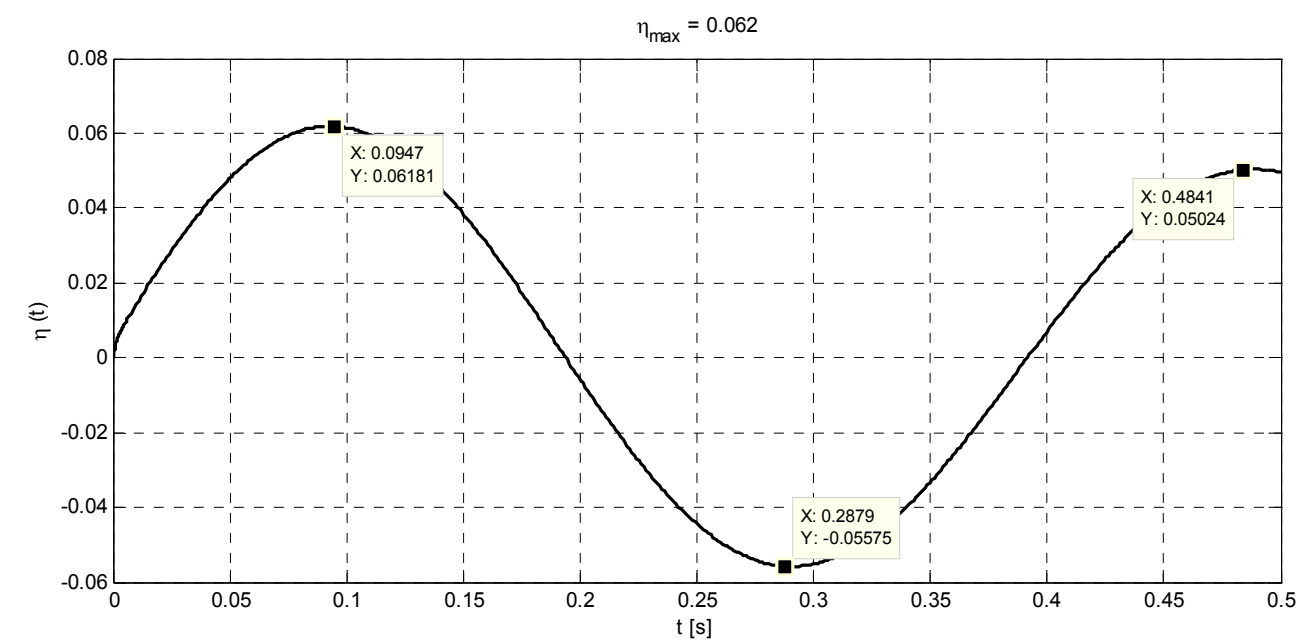

Fig.3. Graph of the function $N_{d}(t)$ and $\eta_{d}(t)$ for a) $m_{k}=0[\mathrm{~kg}]$, b) $m_{k}=1[\mathrm{~kg}]$.

c)
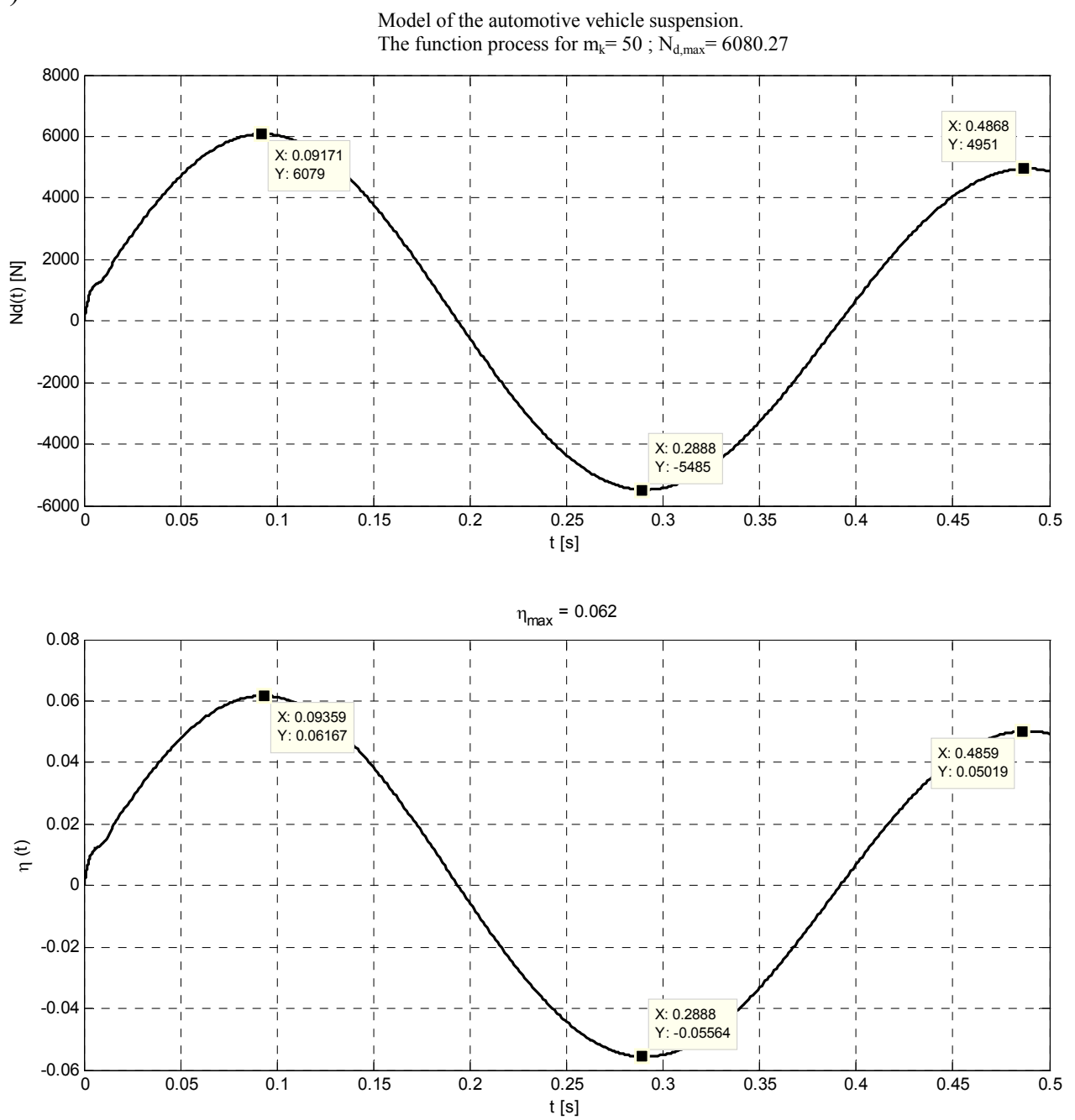
d)

Model of the automotive vehicle suspension.

The function process for $\mathrm{m}_{\mathrm{k}}=100 ; \mathrm{N}_{\mathrm{d}, \max }=6093.94$
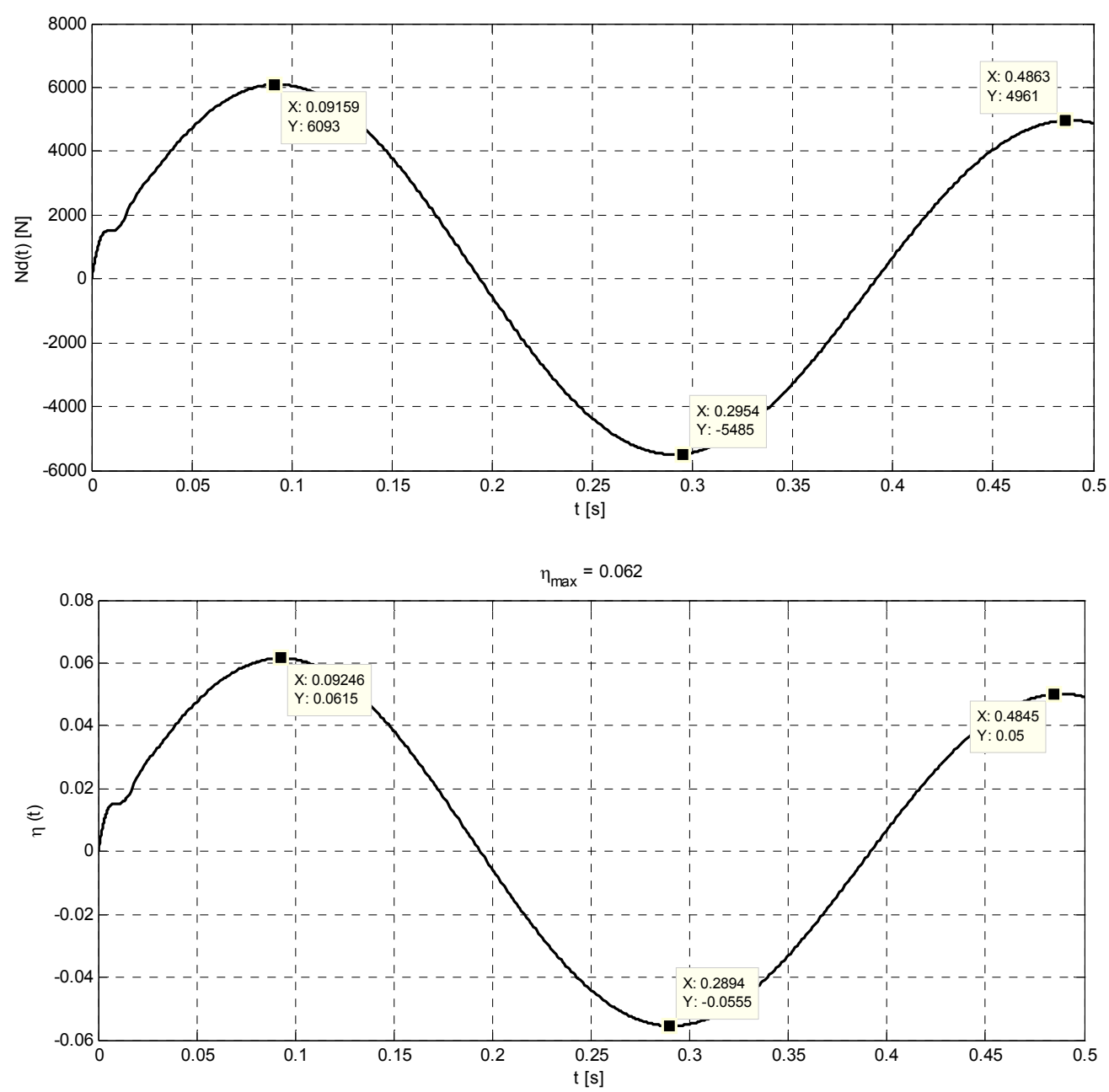

Fig.3. Graph of the function $N_{d}(t)$ and $\eta_{d}(t)$ for c) $m_{k}=50[\mathrm{~kg}]$, d) $m_{k}=100[\mathrm{~kg}]$.

e)

Model of the automotive vehicle suspension.

The function process for $\mathrm{m}_{\mathrm{k}}=200 ; \mathrm{N}_{\mathrm{d}, \max }=6120.80$

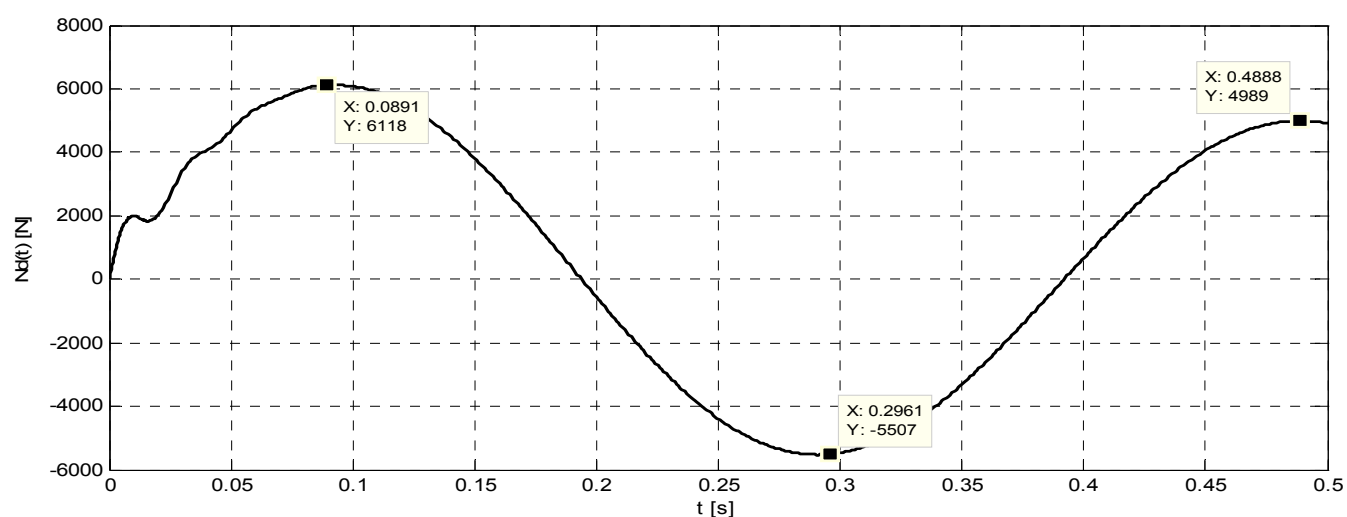




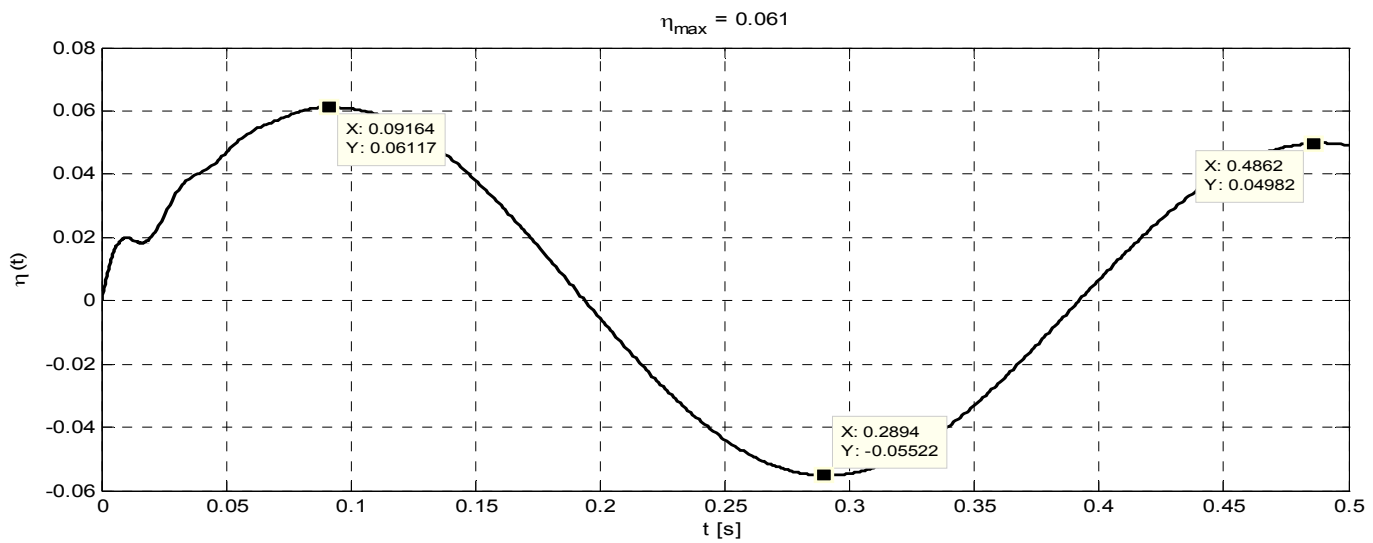

f)

Model of the automotive vehicle suspension.

The function process for $\mathrm{m}_{\mathrm{k}}=500 ; \mathrm{N}_{\mathrm{d}, \max }=6455.75$
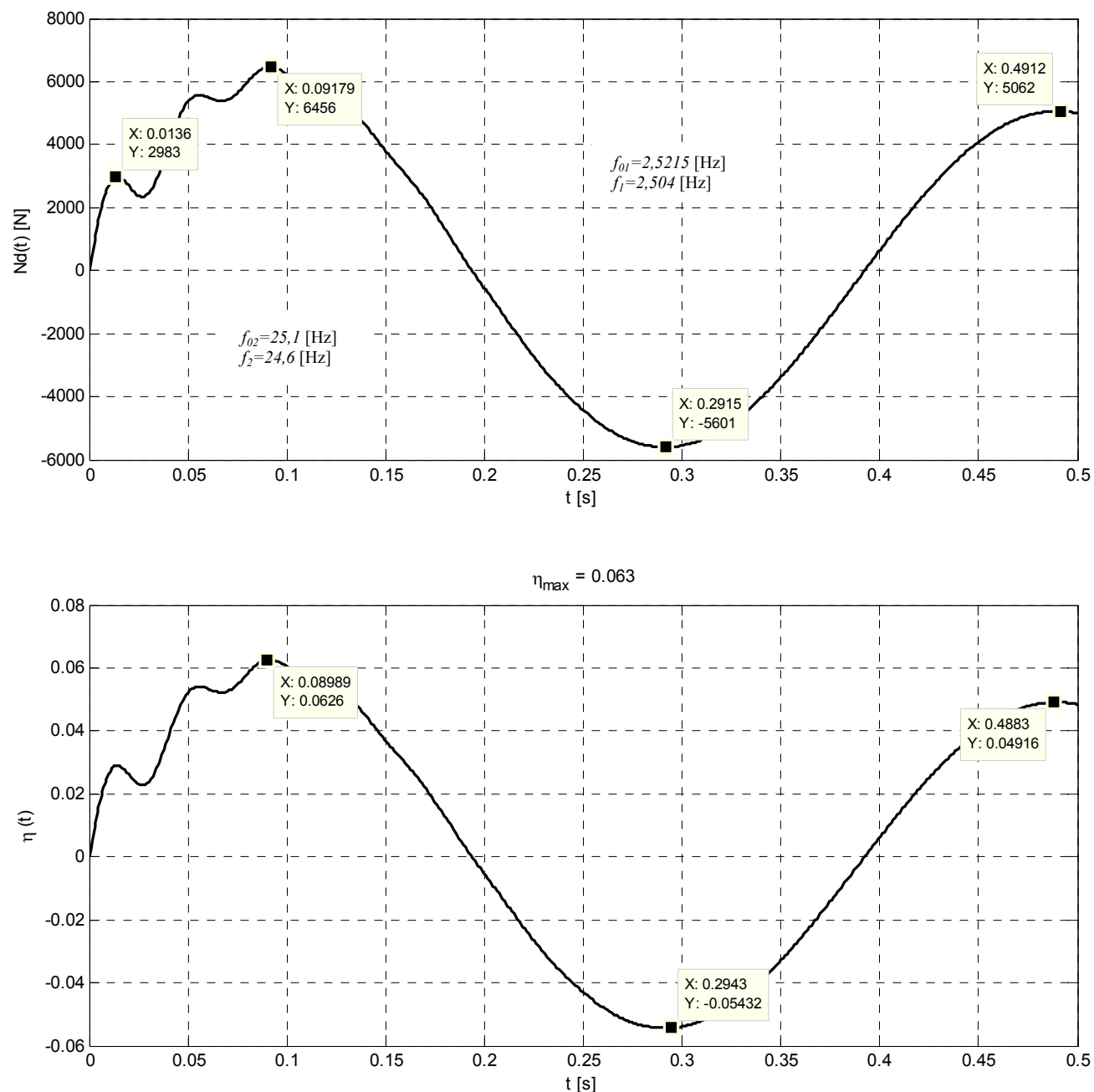

Fig.3. Graph of the function $N_{d}(t)$ and $\eta_{d}(t)$ for e) $m_{k}=200[\mathrm{~kg}]$, f) $m_{k}=500[\mathrm{~kg}]$. 
g)
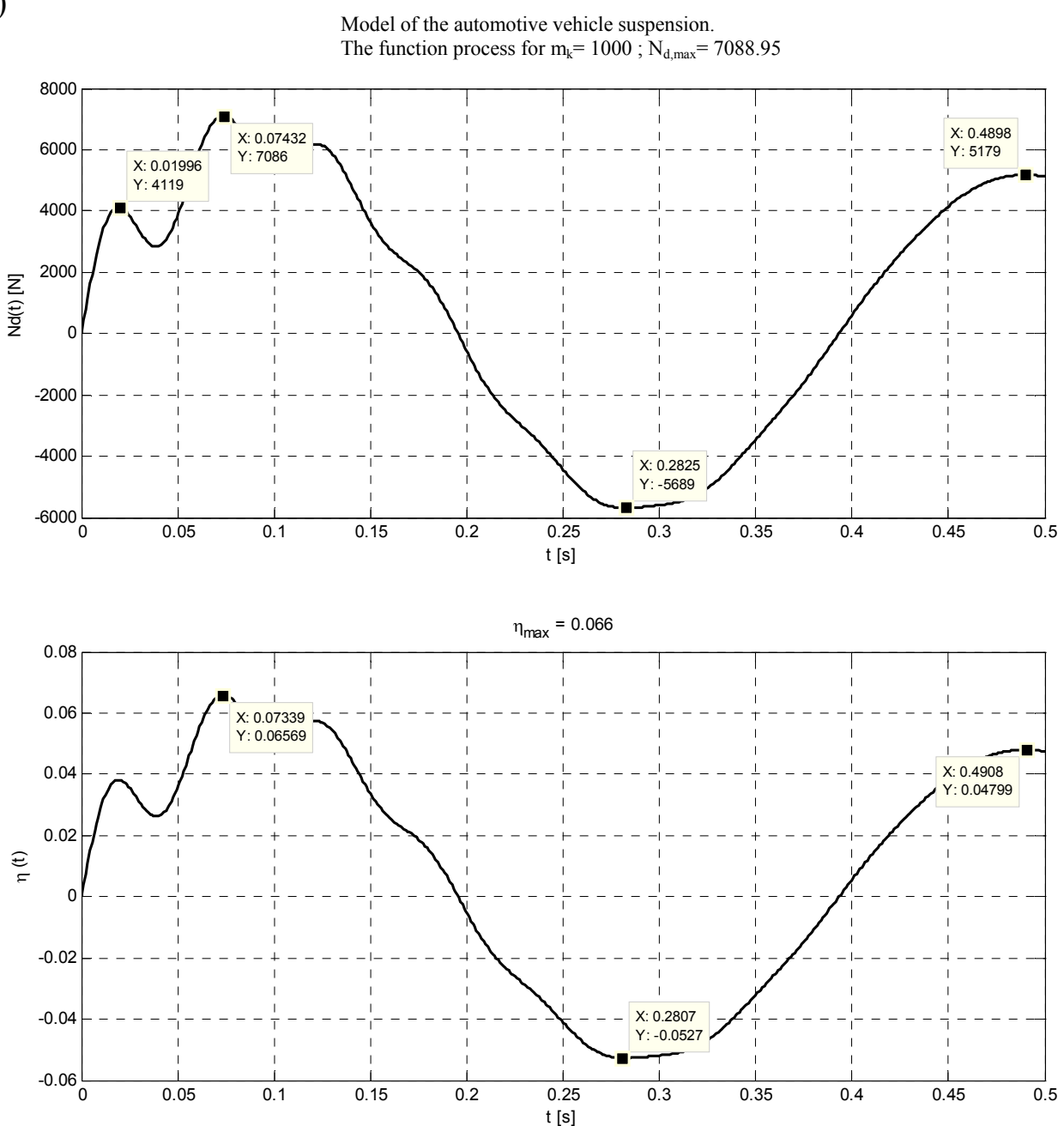

h)

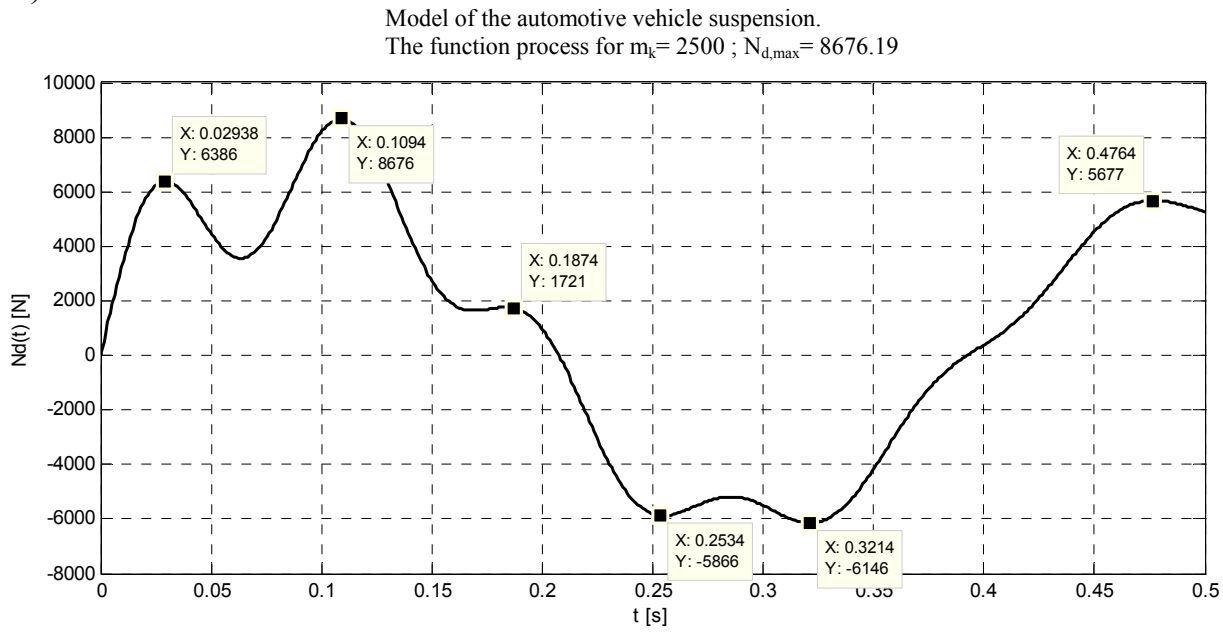




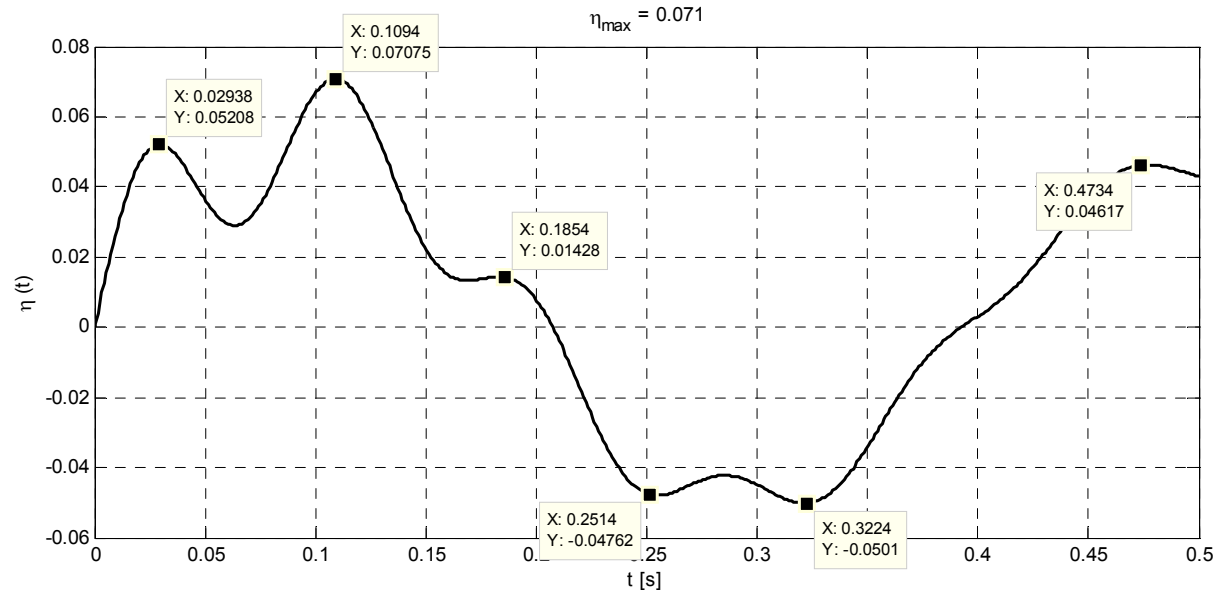

Fig.3. Graph of the function $N_{d}(t)$ and $\eta_{d}(t)$ for $\left.\left.\mathrm{g}\right) m_{k}=1000[\mathrm{~kg}], \mathrm{h}\right) m_{k}=2500[\mathrm{~kg}]$.

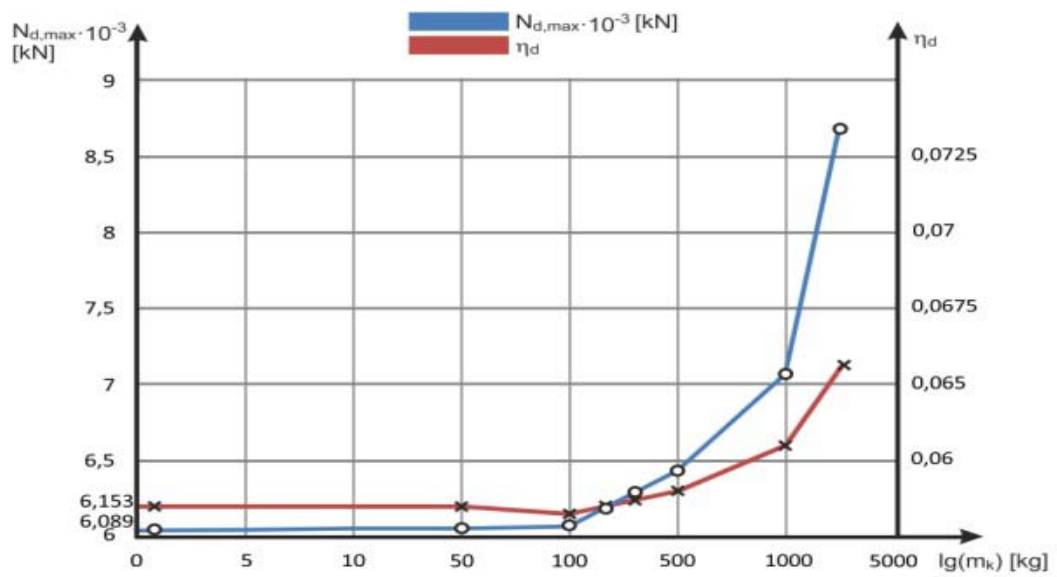

Fig.4. Graph of the function: $N_{d, \max }$ and $\eta_{d}$ depending on $m_{k}$.

Table 1. The calculation results of frequency and coefficients of normal vibration modes as a function of $m_{k}$.

\begin{tabular}{|l|l|l|l|l|l|l|}
\hline$m_{k}[\mathrm{~kg}]$ & $\frac{m_{k}}{m_{n}}[\%]$ & $f_{o, 1}[\mathrm{~Hz}]$ & $f_{o, 2}[\mathrm{~Hz}]$ & $\mu_{1}$ & $\mu_{2}$ & $\mu$ \\
\hline $0(1)$ & $\sim 0$ & 2.5265 & -562.7 & 3.57 & 0 & 1 \\
\hline 50 & 0.5 & 2.526 & 79.58 & 3.56 & $-1.4 e-3$ & 0.9950 \\
\hline 100 & 1.0 & 2.5255 & 56.341 & 3.56 & $-2.8 e-3$ & 0.9900 \\
\hline 200 & 2.0 & 2.5245 & 39.789 & 3.557 & $-5.6 e-3$ & 0.9800 \\
\hline 500 & 5.0 & 2.5215 & 25.1465 & 3.536 & $-14.1 e-3$ & 0.9500 \\
\hline 1000 & 10.0 & 2.51646 & 17.8651 & 3.500 & $-28.6 e-3$ & 0.900 \\
\hline 2500 & 25.0 & 2.501 & 11.3 & 3.395 & $-73.6 e-3$ & 0.750 \\
\hline
\end{tabular}


Table 2. The summary of $N_{d \text {,max }}$ and $\eta_{d}$ values depending on the weight of the axle (wheel) in the range of $<0 ; 2500>$.

\begin{tabular}{|l|l|l|l|l|l|l|l|l|l|}
\hline $\boldsymbol{m}_{\boldsymbol{k}}[\mathrm{kg}]$ & $\mathbf{0}$ & $\mathbf{1}$ & $\mathbf{5 0}$ & $\mathbf{1 0 0}$ & $\mathbf{2 0 0}$ & $\mathbf{3 0 0}$ & $\mathbf{5 0 0}$ & $\mathbf{1 0 0 0}$ & $\mathbf{2 5 0 0}$ \\
\hline $\boldsymbol{N}_{\boldsymbol{d m a x}}[\mathbf{N}]$ & 6066.4 & 6066.8 & 6080.3 & 6093.9 & 6121 & 6153 & 6455.7 & 7088.9 & 8676.2 \\
\hline $\boldsymbol{\eta}_{\boldsymbol{d}}\left(\mathbf{1 x 1 0}^{-3}\right)$ & 62.00 & 62.00 & 62.00 & 61.51 & 61.17 & 60.89 & 63.00 & 66.00 & 71.00 \\
\hline
\end{tabular}

The calculations show that for the value $m_{k} \approx 100[\mathrm{~kg}]$, which is about $1 \%$ of the body weight of the $\mathrm{AV}$ being analyzed, the maximum dynamic force and the dynamic coefficient have an approximately constant value $\left(\eta_{d} \approx 0,062\right)$. When the value of $m_{k}$ is about $200 \mathrm{~kg}$ (approximately $2 \%$ of the body weight), the dynamic coefficient reaches a minimum $\eta_{d, \min } \approx 0.061$.

To verify this result more accurately, additional calculations were performed in the range $m_{k}=<100-350>[\mathrm{kg}]$, changing the value of $m_{k}$ each time by $25[\mathrm{~kg}]$.

The detailed calculation results for $N_{d \text {,max }}$ and the coefficient $\eta_{d}$ in the range of: $m_{k} \in<100 ; 350>$ with the accuracy of $25[\mathrm{~kg}]$ of the reduced axle mass (the accuracy of the calculations $0.25 \%$ ) are tabulated in Tab.3 and shown in the graphs (Fig.5).

Table 3. The values $N_{d, \max }$ and $\eta_{d}$ depending on axle weight (wheel) in the range $<100 ; 350>$.

\begin{tabular}{|c|c|c|c|c|c|c|}
\hline$m_{k}[k g]$ & 100 & 125 & 150 & 175 & 200 & 225 \\
\hline$N_{d \max }[N]$ & 6093.9 & 6101 & 6108 & 6110 & 6121 & 6145 \\
\hline$\eta_{d}\left(1 \times 10^{-3}\right)$ & 61.51 & 61.43 & 61.35 & 61.21 & 61.17 & 61.26 \\
\hline$m_{k}[k g]$ & 250 & 275 & 300 & 325 & 350 & \\
\hline$N_{d \max }[N]$ & 6162 & 6164 & 6153 & 6127 & 6158 & \\
\hline$\eta_{d}\left(1 \times 10^{-3}\right)$ & 61.27 & 61.16 & 60.89 & 60.50 & 60.65 & \\
\hline
\end{tabular}

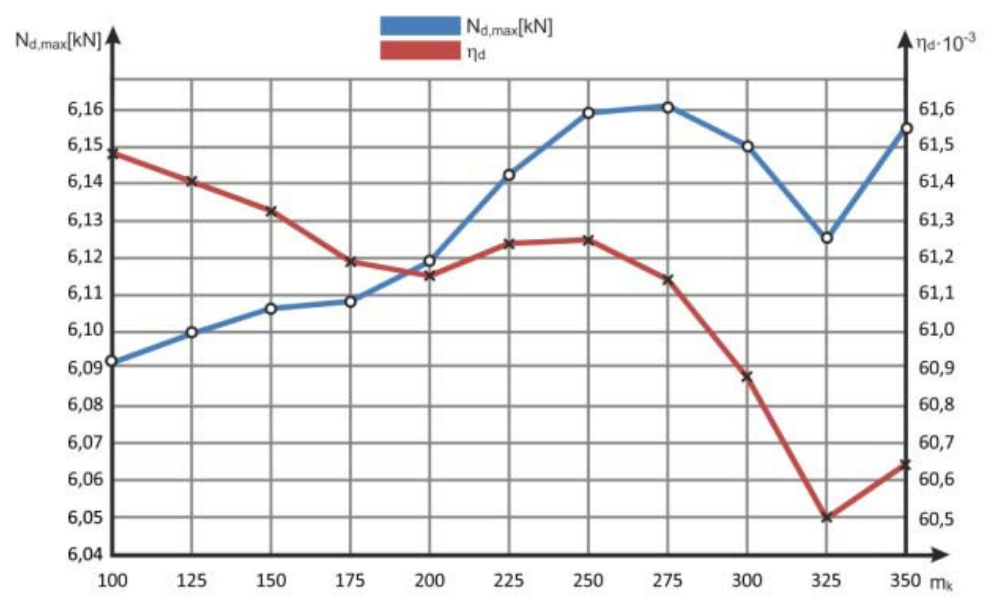

Fig.5. Graph of the function: $N_{d, \max }$ and $\eta_{d}$ depending on $m_{k}$ in the range $<100 ; 350>$.

The calculations show that the force $N_{d \text {,max }}$ comes to a local minimum for $m_{k}=325[\mathrm{~kg}]$ with the accuracy $\pm 25[\mathrm{~kg}]$, while the coefficient $\eta_{d}$ has 2 local minima: for $m_{k(l)}=200[\mathrm{~kg}]$ equal to $\eta_{d(1)}=0.06117$, and $m_{k(2)}=325[\mathrm{~kg}]$ equal to $\eta_{d(2)}=0.06050,[\mathrm{~kg}]$, when $\eta_{d(2)}<\eta_{d(1)}$. After exceeding 
the value $m_{k}>500[\mathrm{~kg}]$, there is a substantial, approximately linear growth both of the force $N_{d \text {, max }}$ and the coefficient $\eta_{d}$. (Fig.5). When the value is $m_{k}=2500[\mathrm{~kg}]\left(25 \%\right.$ of weight of $\left.m_{z}\right) \eta_{d, \max }=0.071$ which is a growth of about $17.4 \%$, relative to the minimum value $(0.06050)$. The static force exerted by the MMP and acting on the axle can be determined by

$$
N_{s t a}=\frac{N_{d, \max }}{\eta_{d}}
$$

On the basis of calculation results (Tab.1), $\eta_{d}=0.0625 \pm 0.005$ was determined for the range $m_{k} \in<50 ; 500>$, which is from $0.5 \%$ to $5 \%$ of the mass $m_{n}$. The average dynamic pressure force on the axle in this range is $N_{d \text {,max,s } r}=6270[N]$. The accuracy of the force measurement can be assumed to be of $\pm 50[N]$. Upon substitution to Eq.(3.1), $N_{s t a, s i r}=100320[N]$ is obtained. The maximum error (for the values assumed above) is $\Delta N_{\text {sta,śr }}= \pm 1610[N]$, which represents approximately $1.6 \%$. The actual static pressure force for $m_{k}=275[\mathrm{~kg}]$ and $m_{n}=10000[\mathrm{~kg}]$ is $N_{\text {sta,rz }}=$ approx. $100798{ }_{[N] \text {, which gives an error }}$ of $N_{\text {sta }}=100320[N]$, approx. $0,5 \%$ in relation to the value determined by the MMP.

\section{Conclusions}

The wheels mass (with axles) does not have much effect on the 1st (fundamental) AV's frequency (Tab.1). The wheels mass has a significant effect on the 2nd frequency (Tab.1). For a given AV (body mass) there is a certain value of the axles mass, for which the dynamic coefficient $\eta_{d}$ reaches a minimum (Fig.4). For larger masses (above $5 \%$ ), the dynamic force acting on the axles increases approximately linearly (and so does the dynamic coefficient) (Fig.3). The dynamic axle load is generated mainly by the 1st frequency (particularly, when the axle mass is small) (Figs 3a-3h). The second self-resonant frequency "is revealed" clearly with heavier axle mass (above $200[\mathrm{~kg}]$, i.e., about $2 \%$ of the body mass. Vibrations having this frequency disappear rapidly due to the damping action of the shock absorber.

\section{Nomenclature}

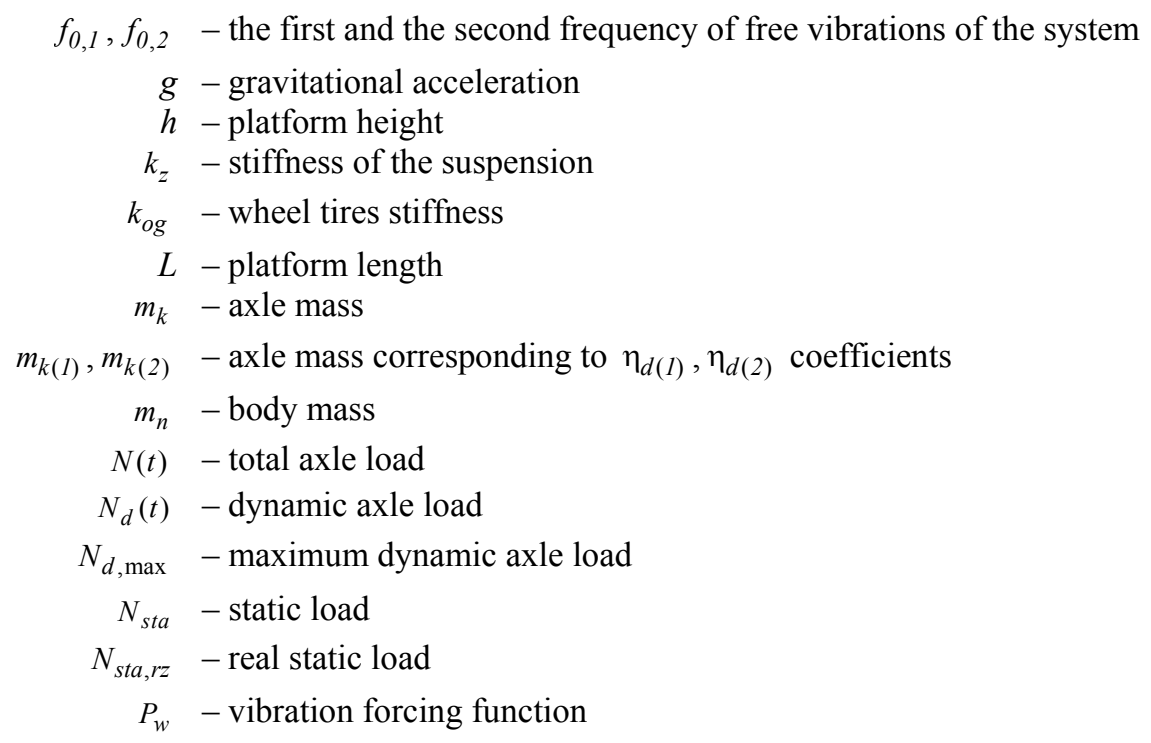


$S_{w}$ - value of impulse of the exciting force

$u(t)$ - lifting displacement

$\dot{u}$ - lifting speed in vertical direction

$V_{k}$ - axle speed in vertical direction

$V_{p}$ - vehicle speed

$V_{u} \quad$ - lifting speed

$z_{k}-$ vertical axle displacement

$z_{n}$ - vertical body displacement

$\dot{z}_{k}$ - axle speed in vertical direction

$\dot{z}_{n}$ - body speed

$\ddot{z}_{k}-$ axle acceleration in vertical direction

$\ddot{z}_{n}$ - body acceleration

$\alpha_{a}$ - shock absorber damping coefficient

$\alpha_{o g}-$ wheel tires damping coefficient

$\eta_{d}-$ dynamic coefficient

$\eta_{d, \text { max }}-$ maximum dynamic coefficient

$\eta_{d(I)}, \eta_{d(2)}-$ dynamic coefficients for the local minimum 1 and 2

$\mu$ - orthogonal coefficient

$\mu_{l}, \mu_{2}-$ coefficients of the first and the second normal vibration modes

$\omega_{0, i}-$ main frequencies of the system

\section{References}

Journal of Laws of the Republic of Poland (2012): No. 1061 of $25^{\text {th }}$ Sept..

Jagiełowicz-Ryznar C. (2013): Dynamic analysis of the vehicle in the movement on the measurement platform. - XII Symposium "Environmental effect of vibrations " Cracow -Wiśnicz.

Kaliski S. (1966): Vibrations and waves in solids. - IPPT PAN, Warsaw.

Mitschke M. (1989): Motor vehicle dynamics. - Vibrations; WKiŁ, vol.2., Warsaw.

Traffic Law Act of $20^{\text {th }}$ June, 1997.

Received: May 12, 2014

Revised: June 22, 2014 